\title{
ZEUXIS Y AZEUXIS EN LA CONFIGURACIÓN SILÁBICA
}

\author{
ZEUXIS AND AZEUXIS IN \\ SYLLABIC CONFIGURATION
}

\author{
ESTEBAN TORRE \\ Universidad de Sevilla
}

\begin{abstract}
Resumen: La agrupación o la separación silábica de dos o más vocales contiguas -entre palabras o en cuerpo de una palabra aislada- da origen a una serie muy compleja de fenómenos fonéticos, que han sido objeto de numerosos estudios, contradictorios en ocasiones. En este trabajo se analizan las distintas circunstancias en las que las vocales en contacto se agrupan en una sola sílaba o, por el contrario, aparecen separadas en sílabas diferentes. Se propone una simplificación terminológica, que consiste en utilizar el término zeuxis para el primer caso, y azeuxis para el segundo.
\end{abstract}

Palabras clave: sílaba, acento, métrica, ritmo, fronteras silábicas, diptongo, hiato.

\begin{abstract}
Syllabic grouping, or separation of two or more adjacent vowels - either between different words or within a single word-, gives rise a very complex series of phonetic phenomena, which have been the object of numerous studies, contradictory at times. This discussion analyses the variable circumstances in which vowels in contact are grouped exclusively within one syllable or, on the contrary, such vowels appear in the form of separate syllables. Here we propose a terminological simplification, which
\end{abstract}


consists of the use of the term zeuxis with regard to the first item, and azeuxis in the second case.

Key words: syllable, accent, metrics, rhythm, syllabic boundaries, diphthong, hiatus. 
$\mathrm{E}$ $\mathrm{N}$ la configuración de la sílaba, como elemento básico del metro, juega un papel de excepcional importancia el contacto entre vocales. En las lenguas románicas, se considera que existe un núcleo, o parte central de la sílaba, que ha de estar siempre representado por una vocal, pudiendo aparecer como partes marginales tanto las vocales como las consonantes. No presenta grandes dificultades la delimitación silábica entre consonantes o entre vocal y consonante, si bien la adscripción de una determinada parte marginal a una u otra sílaba varía según las lenguas y sus distintas convenciones gramaticales. Los problemas surgen cuando la frontera silábica se establece entre vocales. La agrupación o la separación silábica de dos vocales contiguas -entre palabras o en cuerpo de una palabra aisladada origen a una serie muy compleja de fenómenos fonéticos, que han sido objeto de numerosos estudios, contradictorios en ocasiones.

Se ha venido elaborando una extensa terminología con el objeto de distinguir y analizar las distintas circunstancias del encuentro entre vocales. La diversidad de los criterios seguidos y la evolución histórica de los mismos han dado lugar a que algunos términos -diptongo, hiato, sinéresis, diéresis, sinalefa, dialefa, sinafía - no sean utilizados en la actualidad de una manera unívoca por la generalidad de los tratadistas. Como muchas veces ocurre en el dominio de las ciencias humanas, las palabras ahogan las ideas y los términos falsean la realidad. Y, a decir verdad, la realidad que nos ocupa es bien sencilla. Se puede resumir así: las vocales en contacto pueden agruparse en una sola sílaba o, por el contrario, pueden aparecer separadas en sílabas diferentes. En el presente trabajo se propone la utilización del término zeuxis para el primer caso, y azeuxis para el segundo. 
Estos términos y estos conceptos se irán analizando y desglosando en las páginas que siguen.

No se preconiza aquí el olvido de muchos de los términos tradicionales en uso, que pueden ser de utilidad para dar cuenta de los fenómenos fonéticos que entran en juego en determinadas circunstancias. No hay inconveniente alguno en seguir llamando sinalefa a la unión silábica que resulta del contacto entre dos o más vocales pertenecientes a palabras distintas, o sinafía a la sinalefa que se produce entre verso y verso o hemistiquio y hemistiquio. Pero tendríamos que ser precavidos, por ejemplo, a la hora de hacer uso de términos tales como hiato y diptongo en relación con el contacto de las vocales de la serie $/ a, e, o /$ entre sí o con vocales de la serie $/ i, u /$. En el contexto de los estudios gramaticales tradicionales, se ha venido considerando como lo normal y normativo para la formación de diptongos la agrupación de una vocal de las serie $/ i, u /$ con otra de la serie $/ a, e, o /$, o bien la agrupación de las dos vocales de la serie $/ i, u /$ entre sí. El encuentro de las vocales de la serie $/ a, e, o /$ entre sí daría lugar al hiato.

El Esbozo de una nueva gramática de la lengua española (1973) admite ya la posibilidad de que existan diptongos con vocales de la serie /a, e, o/ combinadas entre sí, lo cual había venido siendo antes considerado como anómalo o impropio. Lo cierto es que la división silábica de palabras tales como línea, coágulo, óleo, coexistir no suele ser lí.ne.a, co.á.gu.lo, ó.le.o, co.e.xis.tir, sino lí.nea, coá.gu.lo, ó.leo, coe.xis.tir, esto es, se forman agrupaciones vocálicas que en nada difieren de las consideradas como diptongos.

Así pues, lo habitual es que las palabras línea y óleo, por ejemplo, funcionen como bisílabas. En consecuencia, el uso de la tilde sería innecesario, como no lo es en la palabra santolio. ¿Por qué óleo y petróleo, con tilde, y santolio, sin ella? La razón es muy sencilla: óleo y petróleo se consideran vocablos esdrújulos (de ahí la tilde), y santolio, llano. Es claro que, según los criterios académicos, la intervención de una vocal de la serie cerrada $/ i, u /$ sería imprescindible para la formación «normal» $\mathrm{y}$ «natural» del diptongo. De este modo, vino en denominarse sinéresis (del griego ovvaípeбıs: contracción) el hecho de 
pronunciar en una sola sílaba dos vocales que normativamente deberían formar hiato. Por ejemplo:

Ya los héroes no visten armadura...

Carlos Álvarez

De petróleo y naranja es su arco iris...

Pablo Neruda

En división silábica:

\author{
ya.los.he.roes.no.vis.ten.ar.ma.du.ra \\ de.pe.tro.leoy.na.ran.jaes.suar.co.i.ris
}

En el primer ejemplo, héroes es vocablo bisílabo (he.roes) en virtud de la sinéresis. En el segundo ejemplo, petróleo es trisílabo (pe.tro.leo) por el mismo motivo. Adviértanse también las sinalefas que se originan (leoy, jaes, suar), así como la dialefa (co.i) entre arco e iris.

Más complejo es el caso de la contigüidad de una vocal acentuada de la serie cerrada $/ i, u /$ con una vocal no acentuada de la serie $/ a, e, o /$. Es lo que ocurre, por ejemplo, en las palabras oír, aún, diría. Parece evidente que la formación del hiato es ahora inevitable. Difícilmente se podría admitir un diptongo en el que la vocal nuclear fuera la más cerrada, cumpliendo el papel de elemento marginal la vocal más abierta. Y, sin embargo, en el lenguaje habitual es muy frecuente que una frase como yo diría que... se articule como yo.di.ria.que... Se ha producido, sin duda, una sinéresis ( $i a$ en lugar de i.a). La explicación que suele darse es que ha entrado en juego aquí un mecanismo de dislocación acentual: el acento se habría desplazado de la vocal más cerrada a la vocal más abierta. Pero acudamos, en lo que concierne a las palabras oír y aún, al dominio del verso:

Ver cómo se mece el árbol,

Oír el hilo de la fuente...

JuAN RAMÓN JiMÉNEZ

Aún no nos damos por vencidos. Dicen

Que se perdió una guerra. No sé nada...

Blas de Otero 
En división silábica:

\author{
ver.co.mo.se.me.ceel.ar.bol \\ oir.el.hi.lo.de.la. fuen.te \\ aun.no.nos.da.mos.po.ven.ci.dos.di.cen \\ que.se.per.diou.na.gue.rra.no.se.na.da
}

En el primer ejemplo, hay sinalefa entre mece y el, en este caso entre vocales idénticas. Por otra parte, la primera sílaba de la palabra fuente contiene el diptongo ue, que pertenece al grupo que los criterios normativos contemplan como normal. Además, en este caso, el diptongo proviene de vocal latina y no podría realizarse como hiato, ya que la conciencia lingüística del hispanohablante lo siente como una unidad vocálica indestructible (fuEnte $<$ fontem). En el segundo ejemplo, la sinalefa entre perdió y una da origen al triptongo iou. Pero lo que interesa resaltar aquí es el hecho de tanto oír como aún funcionan como monosílabos. Se da, sin duda, la sinéresis. ¿Pero existe aquí realmente una dislocación acentual? Un buen lector de poesía tal vez perciba el tintineo del hilo de la fuente de Juan Ramón Jiménez en el timbre de la $i$ acentuada del oír, y la rotunda fuerza del aún de Blas de Otero que vibra en esa $u$ profunda y tenebrosa. Veamos, sin embargo, lo que la gramática (Esbozo, párrafo 1.6.8.c) nos dice en un caso similar, esto es, de contacto de una vocal abierta y átona con una vocal cerrada y tónica. En esta ocasión, la unión silábica se da entre palabras, en forma de sinalefa:

Otra curiosa «licencia» poética, que en este caso afecta también a la rima, aparece el endecasílabo de Garcilaso (Égloga I, v. 122): Y por nuevo camino el agua sE Iba, donde se computa como una sílaba el grupo vocálico final /éi/, con dislocación del acento, lo que no impide que iba sea consonante de estiva. Lo mismo ocurre en Góngora (Sonetos completos, ed. 1969, pág. 27): Que a Júpiter ministra el garzón $d E$ Ida, verso aconsonantado en -ida a pesar de la sinalefa /éi/, o en Ponga, pues, fin a las querellas que usa (ibid., 127), con sinalefa /éu/, pero con rima - usa.

El texto citado merece una detenida atención. En primer lugar, es de advertir que los grupos silábicos que se aducen (sei, 
dei, queu) son conceptuados como «sinalefas», y se especifica que se computan «como una sílaba», no que realmente constituyan una auténtica sílaba. Al fin y al cabo, no serían más que curiosas «licencias» poéticas. En segundo lugar, se afirma algo que es obvio: que esas llamadas licencias afectan a la rima. Aquí no hay escapatoria: o existe rima o no existe rima. $\mathrm{Y}$ es innegable que existe la rima: $i b a$, ida, usa. Ahora bien, lo que se entiende por rima no es otra cosa sino la completa igualdad de sonidos con que terminan dos o más palabras a partir de la última vocal acentuada. Por lo tanto, y en pura lógica, si existe la rima (que sí que existe), el acento recae necesariamente en la $i$ de $i b a$, en la $i$ de Ida y en la $u$ de $u s a$. No hay dislocación acentual. Así lo percibían Góngora y Garcilaso. Y así lo percibe el atento y competente lector de poesía. Hay sinalefa, hay diptongo, hay zeuxis, pues así lo demuestra y lo exige la medida del verso. Y hay rima, de acuerdo con la estructura estrófica. En consecuencia, no tiene sentido desplazar el acento a la vocal más abierta. Habría que revisar el concepto de dislocación acentual y admitir que, en casos como éstos, es la vocal más abierta la que se oscurece y se hace menos perceptible, hasta el punto de asumir el papel de mero elemento marginal.

La sinalefa, lejos de ser un recurso excepcional, una licencia métrica o poética, es un fenómeno fonético que se da habitualmente en el verso y en el lenguaje ordinario. Ni los signos de puntuación, ni las pausas que éstos señalan, se oponen a la unión vocálica y silábica que la sinalefa implica. A este respecto, vale la pena considerar la opinión que les merece a los redactores del Esbozo (párrafo 1.6.8.b) la realización de la sinalefa tras una pausa sintáctica:

El verso de metro regular emplea algunas libertades en la formación de la sinalefa. En el endecasílabo de Gerardo Diego (Primera antología, ed. 1947, pág. 62): Mi nombre espera. un día y otro día, la pausa destruye silábicamente el grupo /áu/, pero métricamente se computa como una sílaba, es decir, como una sinalefa. Lo mismo en Bécquer (Rimas, ed. 1911, LXV): ;Estaba en un desierto! Aunque a mi oído. Esta organización del verso es muy frecuente. 
Se termina reconociendo que estamos en presencia de una organización «muy frecuente»; pero, al comienzo del párrafo, se indica ya que se trata de «algunas libertades», rarezas o licencias del verso, y concretamente del «verso de metro regular». Está claro. El verso de metro irregular no presentaría problema alguno, puesto que no está sujeto a la tiranía de las convenciones métricas que obligan al poeta a cosas tan raras -al parecer- como realizar una sinalefa allí donde el oído le dice que tiene que haber sinalefa. Es llamativo el empecinamiento de algunos tratadistas en considerar como excepción, anomalía y rareza aquello que es sencillo y natural para el poeta y para el competente lector de poesía.

Se afirma que la pausa destruye «silábicamente» el grupo /áu/. Es evidente que se confunde la pausa motivada por la sintaxis, por el deseo de destacar el sentido o simplemente por la necesidad de respirar, con la pausa versal, esto es, la pausa final del verso o final de hemistiquio (cesura). No hay otro tipo de pausas en el verso. Ni siquiera la alternancia de hablantes, en la obra de teatro en verso, rompe la continuidad de la línea poética. El Esbozo admite que el grupo /áu/, destruido «silábicamente», puede «métricamente» computarse «como una sílaba». No es ya que no exista realmente una sílaba, ni siquiera una sílaba métrica o fonética, en oposición a las auténticas sílabas gramaticales o fonológicas; no. Lo que ocurre, según el sentir del Esbozo, es que tampoco existiría una verdadera sinalefa, sino sólo que se computa «como una sinalefa».

Serían innumerables las circunstancias que una minuciosa casuística podría establecer en relación con el fenómeno de la sinalefa. Pero, en el fondo, la aparente complejidad de este fenómeno reside más bien en la teoría -0 , por mejor decir, en la elucubración- que en la práctica. Veamos, por vía de ejemplo, el siguiente texto:

¿Qué es aquello que reluce por los altos corredores?

Cierra la puerta, hijo mío; acaban de dar las once.

Federico García Lorca 
En división silábica:

\author{
quees.a.que.llo.que.re.lu.ce \\ por.los.al.tos.co.rre.do.res \\ cie.rra.la.puer.tahi.jo.mi.o \\ a.ca.ban.de.dar.las.on.ce
}

La unión silábica que tiene lugar entre puerta e hijo podría dar lugar a múltiples disquisiciones. El sintagma hijo mio, en función invocativa o apelativa, va justamente entre comas, que obviamente no impiden la sinalefa o zeuxis de ta (de puerta) con hi (de hijo). El problema, para algunos tratadistas, podría residir en el hecho de que el acento de la palabra hijo quedaría anulado en la línea del verso, aunque lo cierto es que dicha palabra conserva de algún modo su independencia acentual: en este caso por obra de la frecuencia acústica, ya que no de la intensidad. Existe, en efecto, un cambio de tono entre puer y tahi que confiere a esta última sílaba una matización acústica - perceptible por audición directa y objetivable en un espectrograma de frecuencias- que viene a suplir la pérdida de intensidad acentual.

La regularidad con que se realiza la sinalefa en la línea del verso, o en la linealidad del discurso ordinario, no impide que alguna vez tenga lugar el fenómeno opuesto: la dialefa. Al no producirse la unión silábica o zeuxis, las vocales que entran en contacto forman hiato o azeuxis:

De la ola, una ola y otra ola, verde mar, verde frío, rama verde, yo no escogí sino una sola ola: la ola indivisible de tu cuerpo.

Pablo Neruda

En división silábica:

\author{
de.la.o.lau.na.o.la. yo.tra.o.la \\ ver.de.mar.ver.de.fri.o.ra.ma.ver.de \\ yo.noes.co.gi.si.nou.na.so.la.o.la \\ la.o.lain.di.vi.si.ble.de.tu.cuer.po
}

En contextos análogos, se pueden dar ya la dialefa, hiato o azeuxis, ya la sinalefa, diptongo o zeuxis: 
Cuerpo de la mujer, río de oro (de.o.ro) donde, hundidos los brazos, recibimos un relámpago azul, unos racimos de luz rasgada en un frondor de oro. (de.o.ro)

cuerpo de la mujer o mar de oro (de.o.ro) donde, amando las manos, no sabemos si los senos son olas, si son remos los brazos, si son alas solas de oro. (deo.ro)

Por otra parte, la acumulación de vocales en hiato - a lo largo de la línea versal o discursiva- ha venido siendo conceptuada, desde el punto de vista retórico, como algo reprobable por su efecto supuestamente desabrido y cacofónico. Habría, por lo tanto, que evitar el hiato. Pero, como ocurre siempre que entran en juego valores estéticos, las cosas no se atienen siempre a los estrictos patrones de la normativa. Veámoslo en un ejemplo:

Ando buscando un verso que supiese parar a un hombre en medio de la calle, un verso en pie - ahi está el detalleque hasta diese la mano y escupiese.

Blas de Otero

En división silábica:

an.do.bus.can.doun.ver.so.que.su.pie.se pa.rar. aun.hom.breen.me.dio.de.la.ca.lle un.ver.soen.pie.a.hi.es.tael.de.ta.lle quehas.ta.die.se.la.ma.no.yes.cu.pie.se

Obsérvese, en la parte central del tercer verso, la cadena de vocales en hiato -/e.a.i.e/- que, lejos de producir un efecto acústico indeseable, restalla como un látigo en la conciencia del lector, adormecido tal vez por la lectura de otro tipo de versos blandos, apagados, relamidos: esos poetas cursis, con sordina,/ hijos de sus papás -nos dirá el mismo autor más adelante-. Se trata de encontrar un verso vivo, un verso en pie, que ande por sí solo: Poetas, perseguid al verso ese,/asidlo bien, blandidlo, y que restalle... 
En lo que concierne al contacto de vocales abiertas de la serie /a,e,o/ con vocales cerradas de la serie /i, $u /$, o de vocales de esta última serie entre sí, se ha venido considerando como normal y natural la formación de diptongos. Pero lo cierto es que, en vocablos como viaje, gradual, cliente, cruel, brioso, sinuoso, diurno, ruido, aunque se haya venido estimando como normativa la agrupación silábica -con diptongos de las formas $i a, u a$, $i e, u e, i o, u o, i u, u i-$ en la pronunciación habitual de estas palabras no se produce el diptongo o agrupación silábica (via.je, gra. dual, clien.te, cruel, brio.so, si.nuo.so, diur.no, rui.do), sino la separación silábica: vi.a.je, gra.du.al, cli.en.te, cru.el, bri.o.so, si.nu.o.so, di.ur.no, ru.i.do. Y es de advertir que en algunas palabras, como ruido, se vacila entre la agrupación (rui.do) y la separación (ru.i.do); en otras, como cruel, lo más frecuente es precisamente la separación silábica (cru.el), al menos en lo que concierne al español peninsular, ya que en la mayor parte de las hablas hispanoamericanas se prefiere el diptongo (cruel).

La preceptiva tradicional consideraba la separación silábica, en todos los casos citados, como una licencia poética o métrica, esto es, como una transgresión de las leyes gramaticales, tolerada solamente en el dominio de la versificación, ya que dichas vocales -por su propia naturaleza- deberían formar diptongo. A la pronunciación de las mismas en sílabas separadas se le dio el

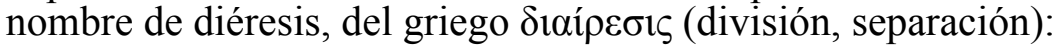

Ya están las rosas primeras dispuestas a embriagarnos.

JUAN RAMÓN JIMÉNEZ

En división silábica: yaes.tan.las.ro.sas.pri.me.ras
dis.pues.tas.aem.bri.a.gar.nos

La lectura del primer verso como octosílabo no ofrece la menor dificultad. Nadie dudaría en realizar la sinalefa entre ya y están. En cambio, en el segundo verso, que también es un octosílabo que forma parte de una serie arromanzada, pueden surgir algunas discrepancias en su escansión. Desde luego, no admite 
discusión la unidad silábica o zeuxis del grupo vocálico ue que aparece en la palabra dispuestas, ya que proviene de vocal latina (dispositas). Pero, en el resto del verso, cabrían dos posibles interpretaciones:

a) Sinalefa entre $a$ y embriagarnos, y diéresis en esta última palabra: aem.bri.a.gar.nos.

b) Dialefa entre $a$ y embriagarnos, y diptongo en esta última palabra: a.em.bria.gar.nos.

Se ha elegido la opción primera, pero no existen razones gramaticales ni explicaciones científicas que avalen esta interpretación. Podríamos aducir, en todo caso, la regularidad con que en la obra de Juan Ramón Jiménez se da la sinéresis en palabras tales como piano, viaje o embriagar. Y hay, naturalmente, motivos estéticos que nos harían rechazar la existencia entre $a$ y embriagarnos de una separación silábica ciertamente desafortunada, que con toda justicia merecería la calificación de hiato, en su acepción etimológica más peyorativa de grieta, resquebrajamiento o bostezo.

Se podría haber llamado a la diéresis simplemente hiato, en oposición a diptongo; pero ese término tenía ya otras acepciones y otros matices retóricos, ya que el hiato (del latín hiatus: hendidura, grieta, abertura, bostezo) implicaría una carga afectiva de cacofonía no deseada. A decir verdad, también el término dip-

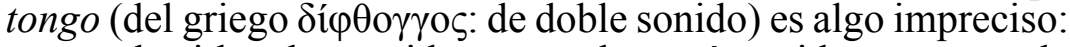
no señala si los dos sonidos o vocales están unidos en una sola sílaba o separados en sílabas diferentes.

En éste, como en todos los casos de vocales en contacto, sería más conveniente hablar de unión o desunión silábica. Y, para ello, la lengua griega nos proporciona dos términos claramente

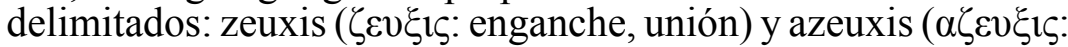

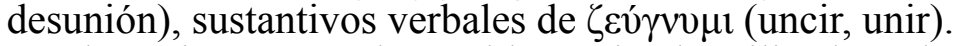

El término azeuxis ha venido ya siendo utilizado en la lengua española, justamente como sinónimo de hiato o encuentro de dos vocales que se pronuncian en sílabas diferentes. No ocurre lo mismo con el término zeuxis, como alternativa a la palabra 
diptongo, pues hasta ahora no ha formado parte de la nomencla-

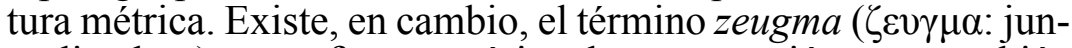
ta, ligadura) como figura retórica de construcción, que también

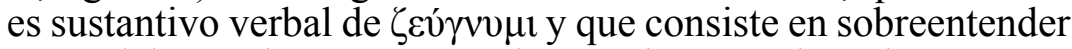
una palabra o sintagma en varias oraciones o cláusulas, aunque sólo está presente en una de ellas. Si aparece en la primera oración o cláusula, se denomina prozeugma o protozeugma. Si aparece en la última, hipozeugma. Y si lo hace en una intermedia, mesozeugma.

Tanto zeuxis como zeugma son, así pues, sustantivos verbales, si bien el primero expresa la acción del verbo, y el segundo, el resultado. Es lo mismo que ocurre con los términos sintaxis ( $\sigma u ́ v \tau \alpha \xi 1 \varsigma:$ orden, disposición, orden de combate) y sintagma ( $\sigma u ́ v \tau \alpha \gamma \mu \alpha:$ ordenamiento, constitución, fila de combate), sustantivos verbales que expresan respectivamente la acción y el resultado de $\sigma v v \tau \dot{\alpha} \sigma \sigma \omega$ (ordenar, organizar). El significado de sintagma como ordenamiento o constitución es patrimonio aún del griego moderno, como puede comprobarse en la denominación de la Plaza de Sindagma (de la Constitución) de Atenas. No es precisamente una lengua muerta la que pervive en la Grecia actual y en otras naciones helénicas, sino un sistema que goza de vigor y lozanía, y que nos sigue ofreciendo vocablos de inestimable valor para la precisa terminología de las ciencias y las artes.

El término zeuxis es más exacto que el diptongo, ya que su significado no se limita a sólo dos elementos fónicos. Pueden ser tres (el llamado triptongo) o más las vocales que se agrupan en una unidad silábica. Del mismo modo, azeuxis carece de las connotaciones peyorativas del hiato, e indica además con toda claridad que existe una desunión de las vocales en contacto, una separación de las sílabas que se constituyen en torno a ellas.

La unión silábica entre palabras, esto es, la llamada sinale-

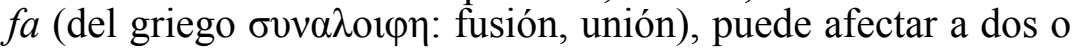
más vocales, originando auténticos diptongos, triptongos y otras agrupaciones vocálicas, a las que es preferible denominar globalmente zeuxis. La separación de las vocales en sílabas diferen-

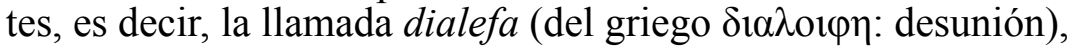
en nada difiere del hiato, o por mejor decir, de la azeuxis. En el 
interior de la palabra, diéresis y sinéresis no son más que realizaciones concretas del hiato y del diptongo, o por mejor decir, de la azeuxis y de la zeuxis.

La unión silábica de la zeuxis y la separación silábica de la azeuxis tienen lugar tanto en la línea sintagmática del verso como en la del lenguaje ordinario, siendo irrelevante que dicha unión o dicha separación se realicen entre palabras o en el interior de la palabra aislada. No parece oportuna la distinción que, en 1973, se hacía en el Esbozo entre sílabas gramaticales y sílabas métricas, o la que, más recientemente, en 2010, se sigue haciendo en la Nueva gramática de la lengua española (1.4ñ) entre sílabas fonológicas y sílabas fonéticas:

Las sílabas fonológicas no se corresponden necesariamente con las sílabas fonéticas. En el verso entre el vivir y el soñar (Machado, Nuevas canciones), se observa la diferencia entre estos dos aspectos. Las sílabas fonológicas en.tre.el.vi.viry.el.so.ñar se convierten desde el punto de vista fonético en en.trel.vi.vir.yel.so.ñar.

Se admite, al menos, que las sílabas «fonéticas» que se producen por el contacto de vocales entre palabras son auténticas «sílabas», sin tener que recurrir al término «sinalefa» para justificar la anomalía originada por la «licencia» poética. Pero se nos dice que, en el citado verso, las sílabas fonológicas «se convierten» en fonéticas, cuando lo que sucede es justamente lo contrario: es la realidad fonética la que se abstrae y se convierte en sistema fonológico. La sílaba presupone el sonido, que es lo que el oído directamente capta. Y conviene poner énfasis en la cualidad acústica, auditiva, del sonido, no en el aspecto meramente oral y articulatorio o en la consideración física de la onda sonora.

La sílaba no tiene en sí misma valor fonológico alguno, a no ser como unidad mínima portadora de un acento. Aunque, a decir verdad, el acento viene a su vez definido, tautológicamente, por un mayor relieve de la sílaba acentuada. La sílaba remite al acento, y el acento a la sílaba. En lo que concierne al contacto entre vocales y su incidencia sobre las posibles divisiones silábicas, carecería de sentido el tratar de atribuir un papel fonológico - distintivo de significados-al hecho de que tenga lugar la zeuxis o la azeuxis. Es obvio que un piano no es más o menos piano, ni 
mejor ni peor, porque tenga dos sílabas (pia.no) o tres (pi.a.no). $\mathrm{Y}$ es que sucede que la sílaba no es un elemento gramatical, sino rítmico.

Sílabas y acentos vienen a configurar el armazón rítmico del verso y su análisis pertenece al dominio de la métrica, la poética y la estética, y no a los estudios gramaticales o lingüísticos. Con buen criterio, la Real Academia Española, que todavía en el Esbozo prestaba alguna atención a las sílabas, el acento y la fonología sintáctica, en la Nueva gramática pasa ya por estas cuestiones como sobre ascuas. Significativamente, el Manual de la Nueva gramática dedica a las unidades fónicas sólo dos brevísimos párrafos, que vienen a ocupar no más de media página de un total de novecientas noventa y tres.

De la sílaba, se nos dice escuetamente que es «el grupo mínimo de sonidos dotado normalmente de estructura interna en la cadena hablada» (Manual, 1.2.1). Conviene que nos detengamos en la calificación adverbial normalmente. Cabría deducir de esa definición que puede haber sílabas anormales o desprovistas de estructura interna. ¿Serán estas sílabas anormales y desestructuradas las que tradicionalmente se consideraban como «métricas», frente a las «gramaticales», y más recientemente «fonéticas», frente a las «fonológicas»?

Si escasa o nula es la atención que el Manual concede a la sílaba, no es mayor la que otorga al acento. En este caso, a la parquedad hay que unir una cierta confusión o una franca incongruencia. Tras hacer alusión a la prosodia como «disciplina que estudia el conjunto de los elementos fónicos SUPRASEGMENTALES», define el acento como «grado de fuerza con el que se pronuncia una sílaba y el que la dota de prominencia con respecto a otras limítrofes» (Manual, 1.2.2).

Conviene recordar que tanto el acento como la prosodia son palabras latinas con idéntico significado: la primera (accentus), como calco del griego $(\pi \rho \circ \sigma \omega \delta 1 \alpha)$; la segunda, como directo préstamo del mismo. En la lengua originaria griega, la prosodia es el canto con acompañamiento de un instrumento musical, el acorde, la armonía, la buena pronunciación. La prosodia, como parte de la gramática, no ha venido significando en efecto otra cosa que pronunciación: pronunciación correcta, pronunciación 
exquisita; del mismo modo que la ortografía se ha venido siempre considerando como correcta escritura, recta grafía. Morfología, sintaxis, prosodia y ortografía eran las partes tradicionales de la gramática. Carece, por tanto, de sentido limitar la prosodia a los elementos fónicos suprasegmentales, esto es, al acento y a la entonación.

Por otra parte, no es oportuno atribuir el acento a la «fuerza» con la que se pronuncia una sílaba. En la constitución del acento -tanto en la palabra aislada, como en la línea discursiva del lenguaje ordinario o en la línea del verso-intervienen fundamentalmente dos cualidades del sonido: la intensidad y el tono. Si atendemos a la intensidad acústica, el acento no es una fuerza, sino una energía, esto es, una magnitud física de mayor complejidad que la fuerza, y proporcional a la amplitud de la onda sonora. Si atendemos al tono musical, entra en juego la frecuencia, es decir, el número de ciclos por segundo de los sonidos emitidos. Y, tanto si el correlato físico del acento es la intensidad, como si lo es el tono, lo verdaderamente importante no es el aspecto suprasegmental, que en la lengua española confiere un valor fonológico a la sílaba acentuada ("cántara", frente a "cantara" o "cantará").

El rendimiento del acento, como unidad suprasegmental, fonológica $-\mathrm{y}$, por lo tanto, distintiva de significados-, es realmente insignificante en español, y nulo en otras lenguas. Lo que de verdad interesa en el acento es el carácter melódico que inserta en la línea discursiva, con su alternancia de sílabas tónicas o más acentuadas y átonas menos acentuadas, lo que origina una especie de pulso o latido rítmico más directamente perceptible que los propios fonemas. Es bien sabido cómo un simple saludo (;buenas tardes!) es más eficaz por la distribución de sus sonidos, sus sílabas y sus acentos que por la materialidad de sus contenidos sémicos.

Sílabas y acentos constituyen el armazón rítmico del verso, la razón de su música. Pero esta música no está superficialmente basada en una fría combinatoria mecánica de unidades abstractas, sino que hunde sus raíces en la viva percepción acústica de la sustancia fónica. Las fronteras silábicas, al igual que la distribución de los acentos en la línea del verso, no obedecen a motivos estrictamente gramaticales. La alternativa entre la unión 
o la separación silábica de las vocales contiguas no depende en ningún caso -si es que hablamos de buena poesía, de auténtica poesía- de caprichosas manipulaciones por parte del poeta, ni de quiméricas licencias artesanales.

Es el sustrato acústico del sonido el que, en aras de la eufonía, une o desune. Y es el oído, que lo capta, el que decide unir o desunir. En el verso con un manso ruido, de Garcilaso de la Vega, se produce la azeuxis (ru.i.do), mientras que en el ruido con que rueda la ronca tempestad, de José de Zorrilla, se realiza la zeuxis (rui.do). En realidad, nada tiene de extraño que el manso ru.i.do se nos presente con la suave lentitud de la separación silábica de la azeuxis, mientras que el abrupto rui.do de la tempestad opte por la rapidez de la unión silábica de la zeuxis, lo que, por otra parte, se compagina muy bien con la sugerencia onomatopéyica del juego aliterativo de las vibrantes múltiples.

En otros casos, no es posible la opción alternativa entre zeuxis y azeuxis, como ocurre en las agrupaciones vocálicas ie y io provenientes de ě y $\breve{o}$ tónicas latinas: bIEn (de bEne), bUEno (de bonum). Aquí se produce siempre la zeuxis. Hasta el día de hoy, en efecto, nadie ha podido pronunciar estas palabras como *bi. en o *bu.e.no. La conciencia lingüística, la intuición y la estética marcan los cauces de la percepción acústica. En los estudios métricos, de nada valdría empeñarse en engrosar la casuística y hacer uso de una terminología cada vez más compleja, dejando a un lado la directa audición de la música del verso. Porque la cosa es bien sencilla: podemos llamar sencillamente zeuxis a la unión silábica de dos o más vocales contiguas, y azeuxis a la separación de las mismas en sílabas diferentes. 